\title{
Prácticas del liderazgo educativo: Una mirada evolutiva e ilustrativa a partir de sus principales marcos, dimensiones e indicadores más representativos
}

\author{
Maureira Cabrera, Óscar Julio \\ dimensiones e indicadores más representativos ${ }^{1}$ \\ Revista Educación, vol. 42, núm. 1, 2018 \\ Universidad de Costa Rica, Costa Rica \\ Disponible en: http://www.redalyc.org/articulo.oa?id=44051918001 \\ DOI: http://dx.doi.org/10.15517/revedu.v42il.22115
}

Prácticas del liderazgo educativo: Una mirada evolutiva e ilustrativa a partir de sus principales marcos,

Esta obra está bajo una Licencia Creative Commons Atribución-NoComercial-SinDerivar 3.0 Internacional. 


\title{
Prácticas del liderazgo educativo: Una mirada evolutiva e ilustrativa a partir de sus principales marcos, dimensiones e indicadores más representativos ${ }^{1}$
}

\author{
Educational Leadership Practices: An Evolutive and Illustrative View From its Main Approaches, Dimensions and \\ More Representative Indicators
}

Óscar Julio Maureira Cabrera ${ }^{2}$

Universidad Católica Silva Henríquez, Chile

DOI: http://dx.doi.org/10.15517/revedu.v42il.22115

omaureir@ucsh.cl

\section{ReSUMEN:}

Diversos y múltiples estudios en esta década han destacado consistentemente, en independencia del contexto sociocultural y modalidades de organización escolar, la relevancia que posee el liderazgo en sus diversas acepciones como factor crítico del desarrollo de las instituciones educativas y sus resultados. Orientado por las interrogantes sobre cuáles son las principales prácticas de los enfoques de referencia en liderazgo escolar en estas últimas tres décadas y qué características evolutivas lo han marcado, se presenta este trabajo de revisión, que con base en el análisis de contenido, describe, ilustra y organiza sistemáticamente, por medio de tablas y figuras, una muestra significativa de dimensiones de la práctica del liderazgo con que se le ha identificado en su evolución conceptual. Como resultado de esto, se destaca que a las prácticas del liderazgo directivo individualista y jerárquicas se le han ido agregando características más participativas y colegiadas asociadas a una mayor distribución del poder en las organizaciones escolares.

Palabras clave: Prácticas de liderazgo, evolución del liderazgo escolar, tendencias de liderazgo educativo, liderazgo distribuido.

\section{ABSTRACT:}

Various and multiple research studies in this decade have consistently highlighted the relevance that leadership has in its different meanings, such as critical factor, independently of the sociocultural context and school organization models, for the development of school and its results. The present study is guided by questions about which the main practices of the approaches of school leadership are in the last three decades, and which evolutive characteristics had an impact on it. Based on content analysis, this work describes, illustrates, and systematically organizes through tables and figures, a significant sample of the dimensions of leadership practice that have been identified in its conceptual evolution. As a result, it is possible to highlight that leadership practices, which are managerial individualistic and hierarchical have been added more participative and collegiate characteristics, associated to a better distribution of power in school organizations.

KEYWORDS: Leadership practice, school leadership evolution, educational leadership tendencies, distributed leadership.

\section{NotAS DE AUTOR}

2 Doctor en educación del Programa Investigación, Diagnóstico y Evaluación en la Intervención Educativa del Departamento de Métodos de Investigación y Diagnóstico en Educación de la Universidad Complutense de Madrid, magíster en gestión educacional y profesor de matemática. Ha trabajado más de 30 años en el sistema educativo escolar chileno como asimismo en su educación terciaria. En su trayectoria ha sido ayudante, becario, docente, académico y directivo en universidades chilenas y española. Ha ocupado cargos de director académico y director de investigación y posgrado en la Universidad Católica Silva Henríquez. Es secretario del Foro Internacional de Innovación Universitaria (FIIU) y miembro del Comité Científico de la Revista Bordón de la Sociedad Española de Pedagogía. Actualmente, es académico titular del Instituto Interdisciplinario de Pedagogía y Educación de la Universidad Católica Silva Henríquez. Sus ámbitos principales de trabajo en formación, investigación e innovación corresponden a dirección y liderazgo en las organizaciones educativas como asimismo gestión de la innovación en la educación terciaria. Sus últimas publicaciones científicas corresponden a: Innovación curricular en la educación superior: ¿Cómo se gestionan las políticas de innovación en los (re)diseños de las carreras de pregrado en Chile?; Más liderazgo distribuido y menos liderazgo directivo: Nuevas perspectivas para caracterizar procesos de influencia en los centros escolares; Liderazgo transformacional en docentes de lenguaje y comunicación en una muestra incidental de establecimientos escolares de vulnerabilidad social. 
La creciente autonomía y responsabilidad por los procesos y resultados en los centros escolares, sustentada tanto por estudios sobre la gestión del cambio para la mejora como por tendencias y prescripciones de política educativa, han potenciado la perspectiva del factor liderazgo en la contribución que este hace al mejoramiento de los aprendizajes, a nivel organizativo escolar. Prueba de ello son los estudios del Programa para la Evaluación Internacional de Alumnos de la OCDE (PISA, por sus siglas en inglés), los que muestran cómo, en el conjunto de países participantes de tal evaluación internacional, cada centro escolar ha ido decidiendo sobre desarrollo curricular, gestión, contratación de profesorado, evaluación docente, entre otros, lo cual ha posibilitado un mayor poder local de decisión en los equipos directivos y más responsabilidad por los resultados obtenidos y comunicación de estos.

El propósito principal de la siguiente revisión consiste en proveer de una mirada global ilustrativa sobre estudio del liderazgo en las organizaciones escolares, con centralidad en sus principales manifestaciones prácticas, tanto para ayudar a su mejor comprensión como también para su eventual transferencia contextual dirigida a la práctica directiva escolar, ya sea para el diagnóstico institucional o para procesos de evaluación de esos aspectos.

Las interrogantes centrales que organizan y guían este trabajo de revisión corresponden a: ¿Cuáles son los principales enfoques, dimensiones e indicadores que se han sustentado como referentes constitutivos de liderazgo en estas últimas tres décadas en las organizaciones escolares? ¿Qué características evolutivas le han marcado? En este sentido, por medio del análisis de contenido de los principales estudios, y a partir de una perspectiva retrospectiva sobre el liderazgo educativo, se describen e ilustran, por medio de tablas y figuras, conceptualizaciones y dimensiones de la práctica del liderazgo en el contexto de los estudios de mejora de la eficacia escolar. Investigaciones que, por lo demás, dieron origen a tal concepto en el contexto de las organizaciones escolares, a raíz del Informe Coleman (Coleman et al, 1966). Posteriormente, se presenta una muestra de dispositivo de transferencia de conocimiento, representada en modelos de evaluación de la calidad de la gestión escolar, aseguramiento y propuesta de estándares, que tienen en su conformación al liderazgo. Finalmente, se expone una serie de interpretaciones sobre la práctica del liderazgo, basada en uno de los enfoques que mayor atención y desarrollo tiene en el campo de la investigación actual sobre el liderazgo escolar, constituido por la distribución del liderazgo.

\section{Breve Retrospectiva de dimensiones práctiCAS de liderazgo EDUCATIVO}

El aporte del factor liderazgo se manifiesta de diversos modos a través del tiempo, así, en un primer momento, las múltiples investigaciones sobre efectividad escolar, impulsadas por las reacciones al Informe Coleman, hace casi medio siglo atrás, señalaban que un liderazgo directivo fuerte, controlador y administrativo constituía la base de cómo se manifestaba este factor para su medición. Se desarrollaron, a comienzos de los años ochenta, instrumentos para medir el liderazgo de directores y directoras, que se refirieron a este como liderazgo directivo. Así, distintos estudios tales como los de Andrews, Soder y Jacoby (1986), Leithwood y Montgomery (1986), Grift (1990), Villanova, Gauthier, Proctor y Shoemaker (1981), realizan las primeras aportaciones a la medida del liderazgo en el campo de la organización escolar.

Una de las escalas más frecuentemente señaladas por la bibliografía, en relación con la dirección escolar es la que desarrolla Hallinger (Murphy y Hallinger, 1984), denominada PIMRS (Principal Instructional Management Rating Scale). Esta escala tuvo como objetivo medir el liderazgo instruccional (pedagógico) de direcciones de centros de educación primaria y secundaria. Las variables que considera este instrumento están basadas en tres dimensiones del liderazgo pedagógico directamente relacionadas con funciones vitales que debiera desempeñar este personal director. En la Tabla 1 se resumen las dimensiones e indicadores de la escala de gestión instruccional creada por Hallinger y Murphy de 1985 (Hallinger, 2008). 
TABLA 1

Marco de liderazgo instruccional (o pedagógico) de Hallinger

\begin{tabular}{|c|c|c|}
\hline Dimensiones & Funciones & Acciones \\
\hline \multirow{2}{*}{$\begin{array}{l}\text { Define la misión de } \\
\text { la escuela }\end{array}$} & $\begin{array}{l}\text { Establece las metas } \\
\text { de la escuela. }\end{array}$ & $\begin{array}{l}\text { Establece metas de lo que es posible lograr con un } \\
\text { propósito en común, y con visión de futuro. }\end{array}$ \\
\hline & $\begin{array}{l}\text { Comunica las metas } \\
\text { de la escuela. }\end{array}$ & $\begin{array}{l}\text { Promueve una cultura escolar que comparte los } \\
\text { valores y creencias de la organización sobre los que } \\
\text { se sustentan los objetivos estratégicos de la escuela. }\end{array}$ \\
\hline \multirow{3}{*}{$\begin{array}{l}\text { Gestiona el } \\
\text { plan curricular- } \\
\text { pedagógico de la } \\
\text { escuela }\end{array}$} & $\begin{array}{l}\text { Gestiona la } \\
\text { contextualización del } \\
\text { currículo. }\end{array}$ & $\begin{array}{l}\text { Prioriza contenidos y desarrollo de habilidades más } \\
\text { relevantes del currículo. }\end{array}$ \\
\hline & $\begin{array}{l}\text { Supervisa y evalúa la } \\
\text { enseñanza. }\end{array}$ & $\begin{array}{l}\text { Recorre las aulas de clases en acuerdo con los } \\
\text { profesores. }\end{array}$ \\
\hline & $\begin{array}{l}\text { Monitorea el } \\
\text { progreso académico } \\
\text { estudiantil. }\end{array}$ & $\begin{array}{l}\text { Planifica la enseñanza con base en el análisis de } \\
\text { datos del progreso de los aprendizajes estudiantiles. }\end{array}$ \\
\hline \multirow{5}{*}{$\begin{array}{l}\text { Fomenta un } \\
\text { adecuado clima de } \\
\text { aprendizaje escolar }\end{array}$} & $\begin{array}{l}\text { Protege el tiempo de } \\
\text { instrucción. }\end{array}$ & $\begin{array}{l}\text { Implementa mecanismo para optimizar el tiempo de } \\
\text { enseñanza en las aulas. }\end{array}$ \\
\hline & $\begin{array}{l}\text { Provee incentivos } \\
\text { para los profesores. }\end{array}$ & $\begin{array}{l}\text { Desarrolla una comunicación efectiva y entrega } \\
\text { retroalimentación al profesorado. }\end{array}$ \\
\hline & $\begin{array}{l}\text { Provee incentivos } \\
\text { para el aprendizaje. }\end{array}$ & $\begin{array}{l}\text { Promueve una cultura de identidad con la escuela, en } \\
\text { que estudiantes y docentes sienten recibir respeto. }\end{array}$ \\
\hline & $\begin{array}{l}\text { Promueve } \\
\text { el desarrollo } \\
\text { profesional. }\end{array}$ & $\begin{array}{l}\text { Desarrolla comunidades } \\
\text { aprendizaje. }\end{array}$ \\
\hline & $\begin{array}{l}\text { Mantiene una alta } \\
\text { visibilidad. }\end{array}$ & $\begin{array}{l}\text { Promueve oportunidades de colaboración con el } \\
\text { profesorado, fomentado un liderazgo compartido. }\end{array}$ \\
\hline
\end{tabular}

Elaborada con base en Hallinger (2012, p.8).

Una de las críticas más fuertes a los modelos de liderazgo instruccional fue que basaban casi toda su influencia en el poder que confería la estructura jerárquica organizativa que mantenía el director o directora del centro. Asimismo, debido a tal predominio del poder posicional, los cambios que perduraban paradójicamente estaban más basados en ideas y acciones que las personas libremente o por convencimiento adoptaban. Es decir, como señala Hallinger (2012), más que posición de poder, el liderazgo instruccional tenía un componente motivacional en docentes y estudiantes, con base en la experiencia y el conocimiento pedagógico de la persona directora.

Como una forma de reacción a las limitaciones del modelo de liderazgo instruccional, las investigaciones se orientaron a modelos de liderazgo que enfatizaran el componente cultural del centro educativo (Lashway, 1995; Sergovianni, 1984). Prueba de ello, es que Leithwood (1994), dentro del contexto del movimiento de reestructuración escolar, adaptaba el modelo de Bass y Avolio (1994) a las organizaciones escolares, denominándolo liderazgo escolar transformacional. Dicho enfoque se basa en atribuciones y habilidades de quien dirige para intervenir en dimensiones de propósitos, personas, estructura y cultura (Maureira, 2004). La Tabla 2 precisa dimensiones, subdimensiones y las correspondientes acciones de tal enfoque de liderazgo transformacional escolar. 
TABLA 2

Dimensiones, subdimensiones y manifestaciones del liderazgo transformacional

\begin{tabular}{|c|c|c|}
\hline Dimensiones & Subdimensiones & Actuaciones más relevantes \\
\hline Propósitos & Visión compartida & $\begin{array}{l}\text { Inicia procesos (periodos de reflexión, etc.) para que el } \\
\text { profesorado se dedique al desarrollo colectivo de una visión } \\
\text { compartida. Adopta una visión para la escuela, pero de forma } \\
\text { que no se "adueña de otras visiones". Clarifica el significado } \\
\text { especíico de la visión de la escuela (o de su propia visión para la } \\
\text { escuela) en lo que se refiere a sus implicaciones prácticas para } \\
\text { los programas, la enseñanza. Utiliza todas las oportunidades a } \\
\text { su alcance para transmitir la visión de la escuela al personal, } \\
\text { estudiantes y familias. }\end{array}$ \\
\hline
\end{tabular}

Demuestra un compromiso infatigable con el bienestar estudiantil. Adopta con frecuencia normas de excelencia. Espera que el personal sea innovador, trabajador y profesional, Expectativas incluyendo estas cualidades entre los criterios que utiliza para contratar nuevo personal. Establece límites muy flexibles para lo que hacen las personas, dándoles libertad de juicio y de acción, dentro del contexto de los planes generales de la escuela.

\begin{tabular}{|c|c|c|}
\hline \multirow{3}{*}{ Personas } & $\begin{array}{c}\text { Apoyo } \\
\text { individualizado }\end{array}$ & $\begin{array}{l}\text { Reconoce el trabajo del personal y da cuenta de ello mediante } \\
\text { el elogio individual. } \\
\text { Su opinión es concreta en lo que se está elogiando como "buen } \\
\text { trabajo". Conoce los intereses del profesorado y a partir de estos } \\
\text { inicia diálogos para el cambio y la innovación. }\end{array}$ \\
\hline & $\begin{array}{c}\text { Estímulo } \\
\text { intelectual }\end{array}$ & $\begin{array}{l}\text { Pone en tela de juicio presunciones básicas del personal sobre } \\
\text { su trabajo, así como las prácticas y creencias inconsistentes } \\
\text { o cuestionables. Anima al personal a experimentar nuevas } \\
\text { prácticas sin utilizar la presión. Busca variadas fuentes de } \\
\text { ideas nuevas, y las comparte con su personal. Visita otras } \\
\text { escuelas en la búsqueda de nuevas ideas. Invita a docentes a } \\
\text { compartir sus competencias con sus colegas. Busca de forma } \\
\text { continua actividades positivas para la escuela e informa e } \\
\text { informa de las que tienen lugar en ella. }\end{array}$ \\
\hline & Modelaje & $\begin{array}{l}\text { Participa decidida y activamente en diferentes actividades } \\
\text { profesionales con docentes, planificando, modelando e } \\
\text { indagando sobre prácticas de liderazgo. Confia en los juicios } \\
\text { del profesorado y entusiasma con su energía para hacer las } \\
\text { cosas lo mejor posible. }\end{array}$ \\
\hline Cultura & & $\begin{array}{l}\text { Fortalece la cultura de la escuela al clarificar la visión de la } \\
\text { escuela para la colaboración del personal docente y para la } \\
\text { atención al estudiantado y el respeto por estos. Comparte con } \\
\text { el personal normas de excelencia, tanto para el personal como } \\
\text { para estudiantes. Utiliza mecanismos para apoyar el trabajo } \\
\text { en colaboración, asignando fondos para crear oportunidades de } \\
\text { colaboración, creando proyectos en que la colaboración sea un } \\
\text { proyecto útil, y contratando personal que comparta la visión de } \\
\text { la escuela, sus normas y valores. Entabla comunicación directa } \\
\text { y frecuente, utilizando todas las oportunidades para dar a } \\
\text { conocer los objetivos de la escuela. }\end{array}$ \\
\hline
\end{tabular}

(Maureira, 2007, p. 155).

Es relevante precisar que, usando este modelo de liderazgo escolar transformacional, Villa (2009) estudió la existencia y magnitud del efecto "cascada" que ejerce este sobre los niveles inferiores de la estructura jerárquica organizacional. Refleja características similares de tales prácticas directivas de liderazgo transformacional en directivos intermedios, tales como jefaturas de estudios o coordinaciones pedagógicas sectoriales. 
Enmarcado en gran parte de las ideas que inspiraron el modelo de liderazgo directivo, Leithwood (2009) generó un modelo de liderazgo directivo cuyas dimensiones y prácticas se resumen en la tabla 3.

TABLA 3

Modelo de liderazgo directivo

\begin{tabular}{|c|c|c|}
\hline Dimensión & Definición & $\begin{array}{l}\text { Prácticas } \\
\end{array}$ \\
\hline \multirow{3}{*}{$\begin{array}{l}\text { Mostrar dirección de } \\
\text { futuro }\end{array}$} & \multirow{3}{*}{$\begin{array}{l}\text { Realizar el esfuerzo de motivar a las } \\
\text { demás personas respecto de su propio } \\
\text { trabajo, estableciendo un propósito } \\
\text { moral. }\end{array}$} & $\begin{array}{l}\text { Construcción de una visión } \\
\text { compartida. }\end{array}$ \\
\hline & & $\begin{array}{l}\text { Fomentar la aceptación de } \\
\text { objetivos grupales. }\end{array}$ \\
\hline & & Altas expectativas. \\
\hline \multirow[b]{3}{*}{ Desarrollar personas } & \multirow{3}{*}{$\begin{array}{l}\text { Construir el conocimiento y las } \\
\text { habilidades que requiere el personal } \\
\text { para realizar las metas de la } \\
\text { organización, así como también } \\
\text { el compromiso y la resiliencia, } \\
\text { disposiciones que este necesita para } \\
\text { continuar realizándolas. }\end{array}$} & $\begin{array}{l}\text { Atención y apoyo individual a } \\
\text { docentes. }\end{array}$ \\
\hline & & Atención y apoyo intelectual. \\
\hline & & $\begin{array}{l}\text { Modelamiento } \\
\text {-interacción permanente y } \\
\text { visibilidad con estudiantes-. }\end{array}$ \\
\hline \multirow{4}{*}{$\begin{array}{l}\text { Rediseñar la } \\
\text { organización }\end{array}$} & \multirow{4}{*}{$\begin{array}{l}\text { Establecer condiciones de trabajo que } \\
\text { le permitan, al personal, el mayor } \\
\text { desarrollo de sus motivaciones y } \\
\text { capacidades. }\end{array}$} & $\begin{array}{l}\text { Construir una cultura } \\
\text { colaborativa. }\end{array}$ \\
\hline & & $\begin{array}{l}\text { Estructurar una organización } \\
\text { que facilite el trabajo. }\end{array}$ \\
\hline & & $\begin{array}{l}\text { Crear una relación productiva } \\
\text { con la familia y la comunidad. }\end{array}$ \\
\hline & & $\begin{array}{l}\text { Conectar a la escuela con su } \\
\text { entorno y sus oportunidades. }\end{array}$ \\
\hline \multirow{4}{*}{$\begin{array}{c}\text { Gestionar la } \\
\text { enseñanza y } \\
\text { aprendizaje en la } \\
\text { escuela }\end{array}$} & \multirow{4}{*}{$\begin{array}{l}\text { Gestión de prácticas asociadas a la sala } \\
\text { de clases y supervisión de lo que ocurre } \\
\text { en el aula. }\end{array}$} & Dotación de personal \\
\hline & & $\begin{array}{l}\text { Proveer apoyo técnico a } \\
\text { los docentes-supervisión, } \\
\text { evaluación y coordinación-. }\end{array}$ \\
\hline & & $\begin{array}{l}\text { Monitoreo de las prácticas } \\
\text { docentes y de los aprendizajes. }\end{array}$ \\
\hline & & $\begin{array}{l}\text { Evitar la distracción del Staff } \\
\text { de lo que es el centro de su } \\
\text { trabajo. }\end{array}$ \\
\hline
\end{tabular}

Elaborado con base en Weinstein y Muñoz (2012, p. 70).

No obstante, dicho modelo ha sido expandido y enriquecido por el Instituto de Educación de Ontario [OISE] a través de un marco que integra, además de las prácticas directivas señaladas en el recuadro, las dimensiones a mejorar: el programa educativo, asegurar la rendición de cuentas y recursos personales de liderazgo. La primera de estas incluye, junto a las asociadas a la dotación de personal, proveer presupuesto, monitorear el progreso del aprendizaje estudiantil y el mejoramiento de la escuela, aislar al personal de distracciones que interfieran con su labor. La segunda, está estructurada a través de la construcción de sentido de rendición de cuentas y el satisfacer las demandas externas de dichas rendiciones. Finalmente, la tercera tiene que ver con los recursos cognitivos, sociales y psicológicos de los líderes formales.

Cabe inferir que a partir del conocimiento producido por el movimiento de investigación (Programa) de mejora de la eficacia escolar y la consistencia del factor liderazgo como clave de efectividad y mejora, se posibilita justificar tal factor como dimensión clave en procesos de evaluación de la calidad de la gestión de los centros. 


\section{El LIDERAZgo EN LOS MODELOS DE EVALUACIÓN DE LA CALIDAD}

Los modelos de evaluación de la calidad de centros educativos que irrumpieron en la década de los noventa, provenientes de las organizaciones productivas no educativas, tuvieron como base la experiencia del mundo empresarial. Tales modelos analizan procesos de gestión y organización de forma más sistemática, organizada y reproducible, es decir, establecen procedimientos que permiten tener una mirada común y estandarizada respecto de qué y cómo mirar las variables que posibilitan evaluar el desarrollo y desempeño de una organización educativa. Por lo tanto, estos modelos describen una visión detallada y precisa de los procesos internos de una escuela y sus modalidades de operación. "Dicho en otras palabras, se ha vuelto necesario contar con modelos e instrumentos que permitan diagnosticar la capacidad de las unidades educativas (Centros Escolares) para producir 'valor agregado' a sus procesos, tanto de enseñanza y aprendizaje como de gestión de ellos” (Alvariño, Arzola, Brunner, Recart y Vizcarra, 2000, p. 3). Así, con diversos énfasis, los modelos tanto de gestión educativa como de aseguramiento de la calidad han destacado al liderazgo formal o directivo como una dimensión clave para impulsar procesos de mejoras educativas.

En la totalidad de los modelos descritos, la dimensión de liderazgo constituye un dinamizante de la gestión en la organización escolar. Tales modelos consideran al liderazgo directivo como clave a la hora de lograr las metas que la organización educativa se plantea. Sin embargo, se visualizan diferencias entre los modelos. Así, por ejemplo, en el caso de Chile, el modelo Sistema de Aseguramiento de la Calidad de la Gestión Escolar (SACGE) tiene como objetivo el mejoramiento progresivo y sistemático de la gestión escolar y las condiciones organizativas para la obtención de niveles de logro educativos. Mientras que el de la certificación de la Fundación Chile, busca desarrollar competencias profesionales entre los actores, con el fin de instalar en la organización educativa "procesos y resultados de calidad". En este sentido, Riveros-Barrera (2012) destaca la relevancia que da gran parte de los modelos de gestión educativa al factor liderazgo, ya que, en estos, tal dimensión se manifiesta en el impulso, poder y competencias que poseen los sujetos directivos escolares para conducir a la mejora continua de sus procesos y resultados, suponiendo la existencia de personal directivo con capacidades de influencia genuinas, lo que, a veces, se confunde con el liderazgo educativo con influencia y control jerárquico de los procesos de la gestión educativa. Asimismo, López (2010, citando a Juste, 2001) precisa que las singularidades de la realidad escolar son re-simplificadas en dichos modelos y que debería analizarse el proyecto educativo como el mayor referente evaluativo del desarrollo escolar.

Desde la perspectiva de las competencias directivas, Robinson, Hohepa, y Lloyd (2009) proponen tres conjuntos fundamentales de características de estas, requeridas para un liderazgo pedagógico efectivo. Así, conocimientos sobre procesos de enseñanza-aprendizaje, vías de influencia para la generación de confianza y resolución de problemas de carácter pedagógico, constituyen el marco competencial del liderazgo pedagógico.

De manera más específica se han generado marcos y propuestas de estándares hacia la función directiva escolar. En este sentido, en el caso de Chile, hace una década la política educativa produjo el Marco para la Buena Dirección (MBD), el cual está siendo renovado y destaca en su nueva propuesta la denominación y conformación del liderazgo como un componente fundamental. La tabla 4 describe las principales prácticas del componente liderazgo en tal marco. 
TABLA 4

Dimensiones de la práctica del componente liderazgo directivo

\begin{tabular}{|c|c|}
\hline Dimensiones & Principales prácticas \\
\hline \multirow{7}{*}{$\begin{array}{l}\text { Liderar los } \\
\text { procesos de } \\
\text { enseñanza y } \\
\text { aprendizaje }\end{array}$} & $\begin{array}{l}\text { Aseguran la articulación y coherencia del currículo con las prácticas de } \\
\text { enseñanza y evaluación como entre los diferentes niveles de enseñanza y } \\
\text { asignaturas. }\end{array}$ \\
\hline & $\begin{array}{l}\text { Identifican y difunden, entre sus docentes y personal directivo, buenas } \\
\text { prácticas de enseñanza y aprendizaje como de gestión pedagógica, tanto } \\
\text { internas como externas y que responden a las necesidades de sus estudiantes. }\end{array}$ \\
\hline & $\begin{array}{l}\text { Monitorean la implementación integral del currículo y los logros de } \\
\text { aprendizaje de todo el estudiantado para el mejoramiento de los procesos de } \\
\text { enseñanza y la gestión pedagógica. }\end{array}$ \\
\hline & $\begin{array}{l}\text { Acompañan, evalúan y retroalimentan sistemáticamente las prácticas de } \\
\text { enseñanza y evaluación del personal docente. }\end{array}$ \\
\hline & $\begin{array}{l}\text { Identifican las fortalezas y debilidades de cada docente de manera de } \\
\text { asignarlo al nivel, asignatura y curso en que pueda alcanzar su mejor } \\
\text { desempeño. }\end{array}$ \\
\hline & $\begin{array}{l}\text { Procuran que sus docentes no se distraigan de los procesos de enseñanza } \\
\text { aprendizaje evitando las interrupciones de clases y la sobrecarga de proyectos } \\
\text { en el establecimiento. }\end{array}$ \\
\hline & $\begin{array}{l}\text { Aseguran la implementación de estrategias para identificar tempranamente } \\
\text { y apoyar al estudiantado que presente dificultades en los aprendizajes o en el } \\
\text { ámbito conductual, afectivo o social. }\end{array}$ \\
\hline
\end{tabular}

Con base en Mineduc (2015, p. 25).

Por otro lado, en un estudio sobre estándares desarrollado por Vera (2016) para el Ministerio de Educación de la República de Chile (Mineduc), en el contexto de las distintas experiencias internacionales sobre direcciones escolares, se precisó que existen siete núcleos temáticos en que se concentraría la labor directiva unipersonal. La tabla 5 muestra dominios y estándares en que concentrarían dichos núcleos temáticos.

TABLA 5

Resumen de estándares sobre el liderazgo directivo escolar

\begin{tabular}{|c|c|c|}
\hline $\begin{array}{l}\text { Núcleo } \\
\text { temático }\end{array}$ & Dominio & Estándar \\
\hline 1 & Pedagógico & Supervisa, influye, orienta la enseñanza y el aprendizaje. \\
\hline 2 & Relacional & $\begin{array}{l}\text { Asegura relaciones adecuadas para el desarrollo de la } \\
\text { organización. }\end{array}$ \\
\hline 3 & Gestión/administración & Forma y desarrolla recursos humanos (instruccional). \\
\hline 4 & $\begin{array}{l}\text { Estratégico } \\
\text { Gestión/Administración }\end{array}$ & $\begin{array}{l}\text { Planifica mejoramiento corto, mediano y largo plazo. } \\
\text { Gestiona recursos materiales. }\end{array}$ \\
\hline 5 & Ético & $\begin{array}{l}\text { Comparte y difunde un éthos organizacional. Creencias, } \\
\text { valores. }\end{array}$ \\
\hline 6 & $\begin{array}{l}\text { De transparencia } \quad y \\
\text { rendición de cuentas }\end{array}$ & Asegura el accountability efectivo de la organización. \\
\hline 7 & Organizacional & $\begin{array}{l}\text { Reestructura la organización, roles y funciones, y } \\
\text { distribuye el liderazgo. }\end{array}$ \\
\hline
\end{tabular}

Con base en Vera (citado por Cuadra, 2014).

Son varias las críticas que desde distintos ámbitos se han generado en torno al concepto de liderazgo directivo. En primer lugar, por su asociación e identificación al mundo de la empresa manufacturera, 
es decir, como concepción que se introduce a un campo de estudio, como es la educación, que difiere sustantivamente en la naturaleza de los procesos y fines provenientes de tal concepto. En segundo lugar, por la reactividad que produce dicho concepto en diversas culturas, debido a sus interpretaciones históricas asociadas a personalidades dictatoriales o políticas que en su afán de poder y dominancia vulneraron derechos humanos fundamentales. En tercer lugar, por la excesiva relevancia que se le da al aporte individual, jerárquico y cupular, en la obtención de notables resultados que se consiguen en la organización, y que omite, entre otros factores, el nivel de recursos disponibles, la calidad de los procesos y la participación de otros actores en decisiones y consecución de dichos logros extraordinarios en tales organizaciones. De hecho, la obra de Bennis (2000), titulada El fin del liderazgo, señala que una economía basada en el conocimiento restringe el individualismo exacerbado y los liderazgos de arriba hacia abajo, precisando que el origen del cambio efectivo se encuentra más en una alianza creativa de la fuerza de trabajo y la alta dirección. Por último, la complejidad del trabajo formativo en las organizaciones escolares requiere, cada vez más, la instalación y desarrollo de comunidades profesionales de aprendizaje, tanto de docentes como de personal directivo.

En este sentido, tiene mucha fuerza, como idea y enfoque de liderazgo, la perspectiva de profundizar y relevar otros componentes en el fenómeno social del liderazgo, como asimismo el resignificar procesos de influencia más genuinos en la contribución de logros en el aprendizaje, tanto organizacionales como estudiantiles. De este modo, la recurrente atención al individuo y su posición de poder en los modelos de evaluación de la calidad de la gestión de los centros, dentro de la estructura jerárquica de la organización, asociada al liderazgo formal (directivo) como único impulsor de la calidad de la gestión de un centro, se comienza a visualizar con dificultades para comprender, en toda su magnitud, los procesos de liderazgo. Se da lugar, así, al resurgimiento de enfoques más participativos y compartidos de liderazgo.

\section{El LIDERAZgo DISTRIBUIDO}

El denominado liderazgo distribuido se ha constituido en un enfoque teórico que enriquece parte de los principios del liderazgo compartido (Senge, 1995) y participativo (Immegart, 1996). Prueba de ello es que sus fundamentos se originan a partir de los principios de las teorías de la cognición distribuida (Hutchins, 2000) y de la actividad (Engerström, 2001).

Los estudios de Gronn (2002) y, luego de Spillane (2006), respecto de la distribución del poder en la organización, han generado una conceptualización focalizada más en la práctica del liderazgo que en la caracterización tradicional de perfiles y roles directivos o de jefaturas, sus antecedentes y consecuencias. Cabe precisar que la acepción líder, desde un contexto distribuido, no se sitúa solo en la figura directiva, sino que se extiende hacia el trabajo de múltiples líderes que ejercen una influencia en las prácticas de enseñanza en la organización escolar. De este modo, el liderazgo como función, se sustentaría en quienes ejercen la dirección, jefaturas intermedias, coordinaciones, profesorado tutor y de aula (Anderson, 2012; Crawford, 2012; Spillane, 2006; Spillane, Diamond, y Jita, 2003).

Sin lugar a dudas, el estudio del liderazgo en las organizaciones escolares no siempre ha considerado a los demás actores formativos y de la comunidad escolar en procesos de influencia relevante, tales como la creación de una base de significados y valores compartidos, la creación de un clima de confianza y apoyo entre docentes que facilite el diálogo y la implicación en un proyecto educativo común. En este sentido, emergen con más fuerza ideas asociadas a los procesos de influencia que consideran los diferentes componentes que lo conforman, resignificándose este fenómeno de estudio más como proceso de influencia social basado en la interacción, que como atribución solo del poder formal de la dirección o jefatura unipersonal. De hecho, algunos estudios refieren a esta perspectiva como liderazgo social (Calvo de Mora, 2011).

Como parte del núcleo clave para comprender la práctica del liderazgo en las organizaciones escolares, Spillane (2006) ha investigado sobre las interacciones que se producen entre líderes, seguidores y situación en 
contextos educativos. En particular, aquellas que tienen directa influencia en la motivación, conocimiento y emociones de integrantes de la comunidad escolar.

Según Spillane y Healey (2010), definir y focalizar de esta manera el liderazgo, excluye prácticas que no están relacionadas con el trabajo esencial de la organización escolar. Una de las cuestiones centrales que plantea Spillane (2006) en torno a su perspectiva distribuida del liderazgo es que este se construye colectivamente para aportar en conocimientos y significados en la organización. Se define como ampliada (líder-plus) y a través de la práctica (Spillane, 2006), a la generada en las interacciones entre líderes, público seguidor y su situación (Spillane, 2006; Spillane, Camburn, Pustejovsky, Stitziel y Lewis, 2008). Desplaza parcialmente la responsabilidad individual-jerárquica al grupo y configura, de este modo, el constructo "líder plus", que se refiere a la acción e interacción conjunta entre quien detenta la autoridad formal como entre quienes emergen como líderes informales en la práctica del liderazgo en un contexto situacional y temporal, tal como se representa en la figura 1.

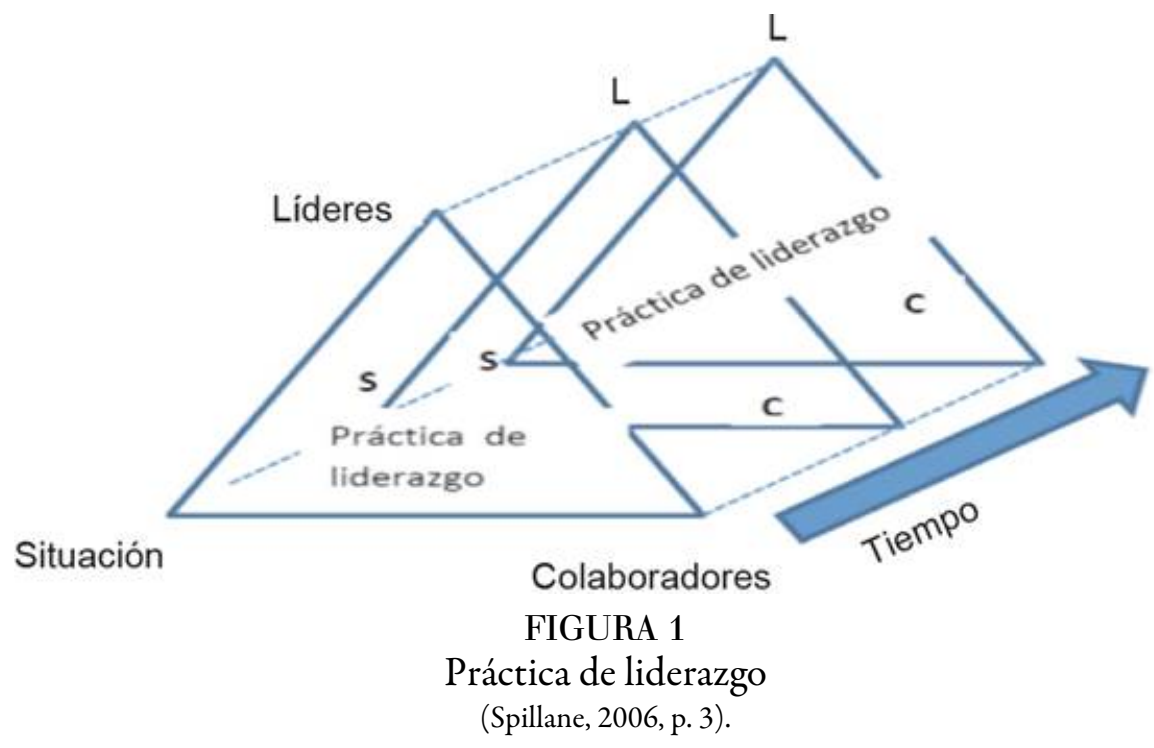

La figura 1 resume gráficamente, por medio de triángulos, que la práctica del liderazgo se da a partir de la interacción en el tiempo de tres componentes fundamentales (vértices del triángulo), como son líderes (formales o informales), situación (práctica socio educativa) y quienes colaboran (determinados por la situación).

Así, según Spillane, Halverson y Diamond (2004), se reconoce que en la práctica del liderazgo hay más de una sola influencia, que esta va en una sola dirección y que una de sus manifestaciones es el compromiso colectivo con la mejora de padres, madres o personas apoderadas (tutores o tutoras), estudiantes, directivos y docentes, la que se refleja en una práctica conjunta y solidaria.

Todo indicaría que la esencia del concepto de liderazgo distribuido, en la perspectiva de Leithwood y Riehl (2005), Murillo (2006), Spillane (2006), y Harris (2009; 2012b), radicaría más en una función de la organización, orientada a distribuir o transferir poder e influencia para la convergencia en propósitos institucionales compartidos, similar a lo que plantea Longo (2008), en el contexto empresarial, sobre la capacidad del personal directivo para transferir, desarrollar y compartir liderazgo. En cambio, Harris (2012a) afirma que el liderazgo es un recurso organizacional estratégico que puede maximizarse, al ocuparse el potencial de influencia que poseen las personas que participan en la comunidad escolar, con especial predominancia de docentes y estudiantes. De este modo, tal estrategia contribuiría a desarrollar e instalar capacidades organizativas en el centro escolar.

Desde una perspectiva más simple, Gronn (2002) refiere al concepto de liderazgo distribuido en dos acepciones. La primera basada en el concepto de desagregación aditiva, es decir, cuántos roles de liderazgo 
existen disponibles en la organización para cumplir los propósitos de esta. Lo que en la práctica significa identificar capacidades de liderazgo individuales que existen en la organización, las cuales consistirían, por un lado, en detectar si los actuales roles de jefaturas y direcciones efectivamente son reconocidos con capacidades de liderazgo o identificar a líderes informales que habitualmente son protagonistas de acciones significativas de cambio y mejora en la comunidad escolar. En cambio, la otra, más holística y compleja, se enmarca en que el foco esencial para visibilizar tal manifestación práctica del liderazgo, el cual estaría situado y contextualizado en una acción coordinada-concertada por un colectivo más que un comportamiento individual agregado.

Desde una perspectiva investigativa, Conger y Pearce (2003) precisan que el liderazgo distribuido puede medirse a través de tres opciones de unidades de análisis. La primera denominada grupal en que el equipo directivo es valorado como un todo en ciertas manifestaciones propias del liderazgo transformacional. Una segunda, como la suma de las partes, es decir, valorar por sí solo el liderazgo de cada integrante del equipo directivo y, finalmente, la tercera, en que al conjunto directivo se le considera como una red social y usa indicadores que miden a cada una de las personas como fuentes de influencia y a cada una de estas como objetivos de influencia.

Por otra parte, Bennet, Wise, Woods y Harvey (2003) sugieren que el liderazgo distribuido es una "manera de pensar acerca del liderazgo" que crítica muchos supuestos sobre este y el tipo de organización dentro de la cual ocurre, consideración que Spillane y Healey (2010) también indican al señalar que el liderazgo distribuido es un lente conceptual para estudiar o diagnosticar el liderazgo y la administración. Sin embargo, el mismo autor, Spillane (citado por López, García, Oliva, Moreta y Bellerín, 2014), precisa que una manera posible en que los centros adquieren una modalidad distribuida de liderazgo es a través del diseño organizativo, es decir, generando cargos que permitan distribuir responsabilidades. Otra forma, por defecto, es que se asumen responsabilidades por cuenta propia cuando se advierte una necesidad. Finalmente, una tercera, es a partir de una crisis institucional.

Una condición clave que posibilita alguna manifestación del liderazgo distribuido es la confianza en la organización. Así, para Day (2013), tal distribución de liderazgo escolar depende de los niveles de confianza que haya en esta, que variarán en un continuo que iría desde la delegación (poca confianza) a la distribución (alta confianza). La siguiente figura propuesta por Day (2013) describe la progresiva distribución de la confianza, base, según él, para un liderazgo exitoso.

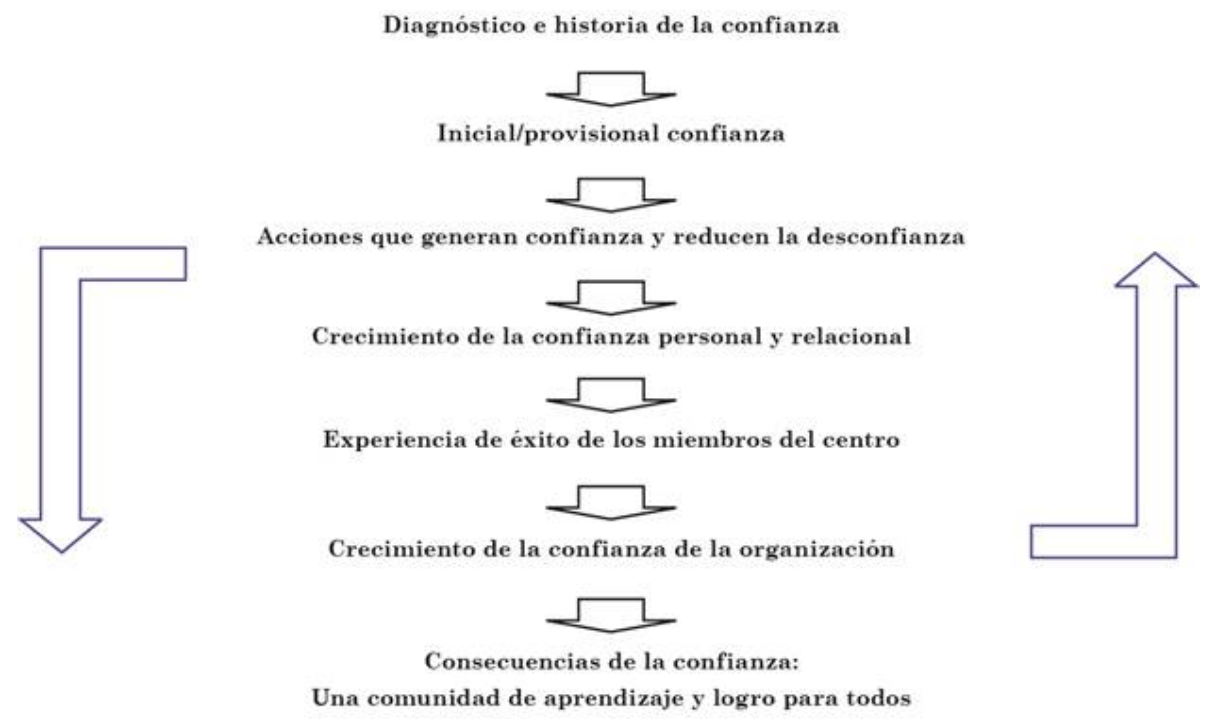

FIGURA 2.

Niveles de distribución de confianzas

(Day, 2013, p.81) 
La figura 2 es una representación que precisa hitos clave del proceso de construcción de confianzas en un centro educativo y de su retroalimentación, a partir del reconocimiento e identificación de su estado inicial o diagnóstico, como asimismo de acciones que se emprenden tanto a nivel personal como relacional para desarrollarla.

Desde la perspectiva de la Organización para la Cooperación y Desarrollo Económico (OCDE), se señala que el liderazgo distribuido es una manera de pensar acerca del liderazgo, promoviendo políticas para la movilización de esfuerzos en que equipos de liderazgo escolar y consejos escolares pueden desempeñar una función vital en el desarrollo escolar (OCDE, 2009). Una dimensión de esta perspectiva se configura a través del área de acción 2, sustentada por la OCDE (2009), como distribución del liderazgo, cuyas prácticas se generarían a partir de los siguientes lineamientos de acción, reflejados en la tabla 6.

TABLA 6

Resumen de áreas de acciones de la distribución del liderazgo

\begin{tabular}{ll}
\hline \multicolumn{1}{c}{ Acciones } & Interrogantes para el diagnóstico y la evaluación \\
\hline $\begin{array}{l}\text { Reducir la carga de directores/as de } \\
\text { escuela }\end{array}$ & $\begin{array}{l}\text { ¿Qué contribución pueden hacer otros entes para reducir la } \\
\text { carga de liderazgo escolar de directores/as en su contexto? }\end{array}$ \\
\hline $\begin{array}{ll}\text { Distribuir el liderazgo para la eficacia } \\
\text { escolar }\end{array}$ & $\begin{array}{l}\text { ¿Qué incentivos existen en su sistema para animar a las } \\
\text { personas a participar en equipos de liderazgo distribuido? }\end{array}$ \\
\hline $\begin{array}{ll}\text { Apoyar a los consejos escolares en el } \\
\text { desempeño de su función }\end{array}$ & $\begin{array}{l}\text { ¿Qué medidas se toman actualmente en su contexto para } \\
\text { asegurar que los consejos escolares sean representativos y } \\
\text { demuestren un nivelapropiadodehabilidady compromiso? }\end{array}$ \\
\hline $\begin{array}{ll}\text { Reflejar el liderazgo distribuido en la } \\
\text { politica }\end{array}$ & $\begin{array}{l}\text { ¿Hasta qué grado la política existente (por ejemplo, } \\
\text { relacionada con la rendición de cuentas) apoya u } \\
\text { obstaculiza el liderazgo distribuido? }\end{array}$ \\
\hline $\begin{array}{ll}\text { Sostenimientodelliderazgodistribuido } \\
\text { ¿Qué retos y oportunidades en las escuelas pueden ser } \\
\text { abordados con mayor productividad por equipos de } \\
\text { liderazgo distribuido? }\end{array}$ \\
\hline
\end{tabular}

Liderazgo distribuido escolar (OCDE, 2009, p. 18)

Otra perspectiva de la práctica del liderazgo distribuido, según Spillane (citado por Riveros-Barrera, 2012), señala que cuando el liderazgo se entiende como una propiedad de la escuela, este se puede deducir de las interacciones producidas que existen, generándose modalidades de distribución de liderazgo. A saber: la colaborativa, que estaría enmarcada en interacciones en que trabajan dos o más individuos simultáneamente en tiempos y espacios. La colectiva, que sería aquella interacción en que trabajan de manera interdependiente, pero en tiempos y espacios distintos, y una tercera, denominada coordinada, cuya interacción está basada en la secuencialidad de acciones. La siguiente tabla 7 resume prácticas en que se ven reflejada tales situaciones. 
TABLA 7

Modalidades de prácticas de liderazgo distribuido

\begin{tabular}{|c|c|c|}
\hline & Colaborativa & Coordinada \\
\hline $\begin{array}{l}\text { División } \\
\text { del } \\
\text { Trabajo }\end{array}$ & $\begin{array}{l}\text { Distintos líderes formales } \\
\text { son responsables de una } \\
\text { parte de un proceso o } \\
\text { estrategia e interactúan } \\
\text { con equipos colaboradores } \\
\text { en la escuela durante el } \\
\text { año lectivo para alcanzar } \\
\text { los resultados o productos } \\
\text { esperados. }\end{array}$ & $\begin{array}{l}\text { Distintoslíderesformales } \\
\text { son restintos líderes formales son } \\
\text { de una parte de un de un proceso o estrategia y } \\
\text { proceso o estrategia y distribuyen tareas a otras } \\
\text { delegan tareas a otras personas miembros de la } \\
\text { personas miembros organización cuyos productos } \\
\text { de la organización son prerrequisitos de otros } \\
\text { cuyos productos se para alcanzar los resultados } \\
\text { correlacionan para esperados. } \\
\text { alcanzar los resultados } \\
\text { esperados. }\end{array}$ \\
\hline $\begin{array}{l}\text { Co- } \\
\text { liderazgo }\end{array}$ & $\begin{array}{l}\text { Distintos líderes } \\
\text { formales comparten la } \\
\text { responsabilidad sobre } \\
\text { un mismo proceso o } \\
\text { estrategia alternándose } \\
\text { para conducir la actividad } \\
\text { e interactuando con } \\
\text { equipos, colaboradores en } \\
\text { la escuela durante el año } \\
\text { lectivo para alcanzar los } \\
\text { resultados o productos } \\
\text { esperados. }\end{array}$ & $\begin{array}{l}\text { Distintos líderes } \\
\text { formales comparten la formales comparten la } \\
\text { responsabilidad sobre responsabilidad sobre un } \\
\text { un mismo proceso o mismo proceso o estrategia } \\
\text { estrategia alternándose alternándose para conducir } \\
\text { para conducir la actividad la actividad y distribuyendo } \\
\text { y delegando tareas a tareas a otras personas } \\
\text { otras personas miembros miembros dela organización } \\
\text { de la organización cuyos cuyos productos son } \\
\text { productos se correlacionan prerrequisitos de otros para } \\
\text { para alcanzar los alcanzar los resultados } \\
\text { resultados esperados. }\end{array}$ \\
\hline $\begin{array}{l}\text { Liderazgo } \\
\text { paralelo }\end{array}$ & $\begin{array}{l}\text { Distintos lideres formales } \\
\text { son responsables de áreas } \\
\text { de trabajo diferentes de } \\
\text { similar relevancia en } \\
\text { la organización y cada } \\
\text { quien interactúa con } \\
\text { equipos colaboradores en } \\
\text { la escuela durante el año } \\
\text { lectivo para alcanzar los } \\
\text { resultados o productos } \\
\text { esperados. }\end{array}$ & $\begin{array}{l}\text { Distintos lideres formales Distintos lideres formales } \\
\text { son responsables de áreas son responsables de áreas } \\
\text { de trabajo diferentes de trabajo diferentes } \\
\text { de similar relevancia de similar relevancia } \\
\text { en la organización y en la organización y } \\
\text { delegan tareas a otras distribuyen tareas a } \\
\text { personas miembros de otras personas miembros } \\
\text { la organización, cuyos de la organización } \\
\text { productos se correlacionan cuyos productos son } \\
\text { paraalcanzarlosresultados prerrequisitosdeotrospara } \\
\text { esperados. } \\
\text { alcanzar los resultados } \\
\text { esperados. }\end{array}$ \\
\hline
\end{tabular}

Perspectivas de la práctica del liderazgo distribuido (Vera, 2016, p.21).

Por su parte, el trabajo de Macbeath (2011), representado en la figura 3, deriva en seis formas de distribución, que se caracterizan como una taxonomía o continuo para sugerir un flujo entre ellos, pero también con el fin de señalar su carácter situacional. Aunque cada una de estas diferentes manifestaciones de liderazgo puede ser apropiada en un momento y contexto dado, el liderazgo de mayor éxito sería, a nuestro juicio, transmitir una comprensión de todas estas diferentes expresiones de la distribución y ser capaz de operar en cada modo según la situación y el estado de desarrollo de la escuela, con el objeto de alcanzar la distribución cultural o lo que se asimila a lo que algunos estudios han denominado la cultura o ecología del liderazgo. 


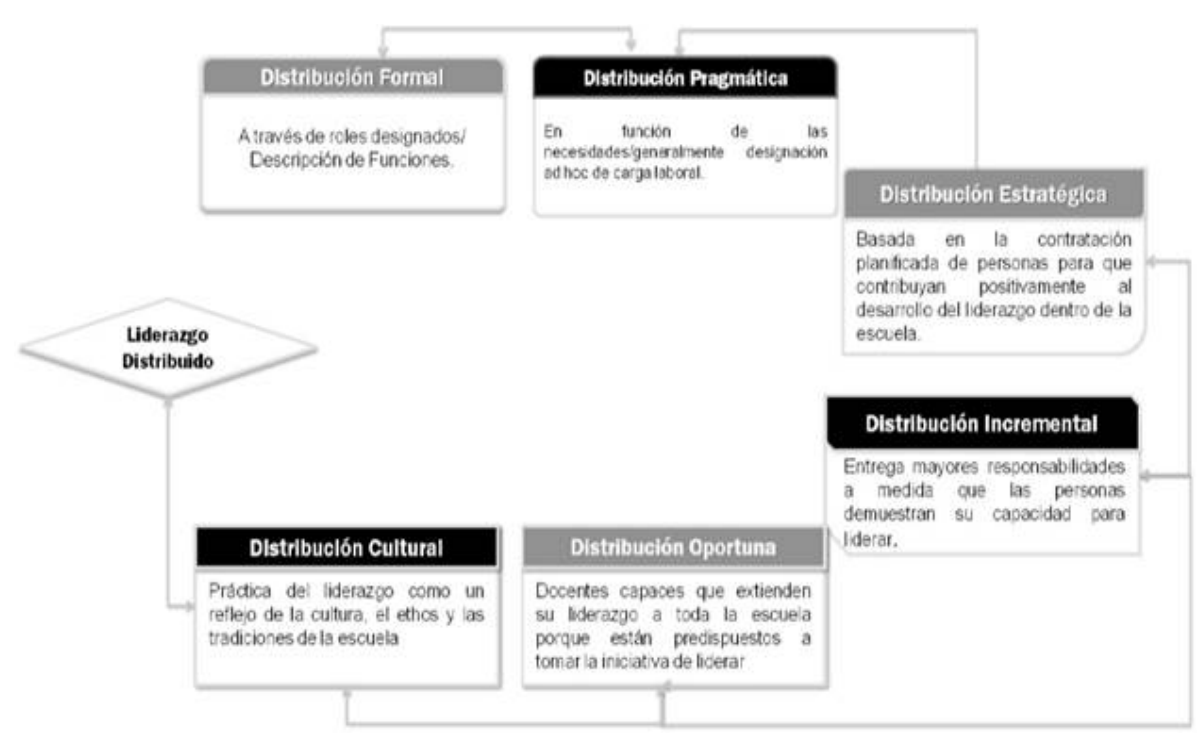

FIGURA 3.

Estadios de distribución del liderazgo

(MacBeath, 2011, p. 94).

La figura 3 caracteriza, en un continuo de seis distinciones, con sus correspondientes definiciones, la concepción de distribución de liderazgo de un centro educativo. Así, en tal figura se reconoce la descripción de funciones y la asignación de cargos mediante esta, como una primera manifestación de la distribución del liderazgo, denominada formal. Seguidamente, la distribución pragmática, estratégica, incremental y oportuna significa matices intermedios de la distribución de tal liderazgo. Finalmente, como un estadio de desarrollo deseable se configura la distribución del liderazgo como parte de la cultura organizacional del centro.

Desde otra perspectiva, Harris (citado por López et al., 2014), caracteriza dos factores independientes en relación con la práctica de la distribución del liderazgo, el primero asociado al alcance de la distribución del liderazgo (difusa / profunda) y otro, sobre el nivel de acople organizativo (débil / fuerte). La tabla 8 resume y describe tales prácticas distribuidas.

TABLA 8

Práctica distribuida de liderazgo

\begin{tabular}{|c|c|c|c|}
\hline & \multicolumn{3}{|c|}{ Alcance de la distribución } \\
\hline & & Difusa & Profunda \\
\hline \multirow{2}{*}{$\begin{array}{c}\text { Acople } \\
\text { organizativo }\end{array}$} & Débil & $\begin{array}{l}\text { Distribución ad hoc } \\
\text { Práctica descoordinada en una } \\
\text { estructura flexible }\end{array}$ & $\begin{array}{l}\text { Distribución autónoma } \\
\text { Práctica coordinada en una } \\
\text { estructura flexible }\end{array}$ \\
\hline & Fuerte & $\begin{array}{l}\text { Distribución autocrática } \\
\text { Práctica esporádica en una } \\
\text { estructura rígida }\end{array}$ & $\begin{array}{l}\text { Distribución aditiva } \\
\text { Prácticas limitadas, aunque } \\
\text { coordinadas en una estructu- } \\
\text { ra rígida }\end{array}$ \\
\hline
\end{tabular}

Un modelo de práctica distribuida de liderazgo (adaptado de Harris, 2008) en López (2013, p. 38).

Finalmente, desde lo más macro político, el liderazgo distribuido ha sido caracterizado como un esfuerzo de coordinación de directores y directoras, de un determinado distrito local o comuna, a objeto de compartir diferentes prácticas directivas que llevan a cabo en sus contextos escolares y desarrollar estrategias de 
aprendizaje para establecerse como una comunidad profesional de aprendizajes directiva. Tal manifestación del liderazgo distribuido ha sido interpretada como una estrategia de gestión del conocimiento de prácticas directivas. La tabla 9 resume algunas experiencias internacionales.

TABLA 9

Concepciones de prácticas local o del meso sistema escolar del liderazgo distribuido

\begin{tabular}{|c|c|c|}
\hline Pais & Denominación & Principales prácticas \\
\hline Finlandia & Liderazgo sistémico & $\begin{array}{l}\text { Redistribución del liderazgo al interior de los municipios, entre } \\
\text { autoridades municipales y escolares, entre escuelas y dentro } \\
\text { de estas mismas, introduce cambios significativos en el sistema } \\
\text { local: aumentan las interacciones entre actores, fortalece una } \\
\text { mutua interdependencia, mejora las comunicaciones, y hace } \\
\text { más permeables los limites organizacionales al aumentar las } \\
\text { capacidades para resolver problemas y generar espacios para } \\
\text { desarrollos futuros. }\end{array}$ \\
\hline Canadá & $\begin{array}{l}\text { Liderazgo } \\
\text { distribuido en aras } \\
\text { de escuelas más } \\
\text { inteligentes }\end{array}$ & $\begin{array}{l}\text { Conjunto de prácticas para fijar rumbos y ejercer influencia, } \\
\text { potencialmente por personas de todos los niveles, más que un } \\
\text { conjunto de características personales y atributos de quienes } \\
\text { están en la cúspide organizacional. }\end{array}$ \\
\hline Australia & $\begin{array}{l}\text { Marco de desarrollo } \\
\text { y aprendizaje para } \\
\text { el liderazgo }\end{array}$ & $\begin{array}{l}\text { Cultura de liderazgo en todo el sistema, basada en un } \\
\text { discurso profesional con un lenguaje común. Conversación del } \\
\text { mejoramiento escolar, el desarrollo de liderazgo se promueve } \\
\text { y trasmite en todo el sistema a través de todas las secciones de } \\
\text { la infraestructura educativa. Clara visión de características } \\
\text { del liderazgo efectivo y aprendizaje evolutivo. }\end{array}$ \\
\hline
\end{tabular}

Experiencias de liderazgo distribuido en una muestra de países de la OCDE (Maureira et al., 2014, p. 146).

\section{A MODO DE CIERRE}

Si bien es bastante y variada la bibliografía sobre el liderazgo en las organizaciones escolares en estas últimas décadas, dentro del contexto iberoamericano, no siempre su contenido ha estado situado en describir y destacar de manera ilustrativa, resumida y operacional cómo se infiere y manifiesta la práctica de este. En este sentido, el trabajo de revisión expuesto se ha centrado en visibilizar este componente a través de una muestra significativa de enfoques, dimensiones e indicadores de realización de las principales perspectivas de liderazgo escolar.

En sus inicios, el liderazgo escolar, traducido desde el inglés como instruccional, fue comprendido desde la práctica como un ejercicio exclusivo del dirección y sus principales manifestaciones se centraron en componentes más administrativo-burocráticos que inspiracionales y motivacionales. Sin embargo, al analizar los distintos enfoques y sus respectivas dimensiones, se observa a través del tiempo el enriquecimiento en sus componentes de visión y su engrandecimiento con otras, destaca en las que destaca el modelo de Hallinger (2012) como uno de los referentes más importantes para enmarcar tal liderazgo directivo pedagógico y curricular. No obstante, sus manifestaciones prácticas están más situadas en la enseñanza que en asegurar un aprendizaje efectivo en todo el estudiantado del centro escolar, dimensión que sí está presente en las perspectivas de liderazgo de Elmore (2010) y Robinson et al. (2009).

Con foco en lo organizacional y como aplicación al campo de las organizaciones educativas, Bass y Avolio (1994) enriquecen sus diferentes estudios sobre liderazgo en distintos tipos de organizaciones no educativas y prueban similares hipótesis en otro tipo de instituciones, con lo que generan un conjunto de investigaciones que promueven el modelo de liderazgo transformacional de rango total. Así, con estos hallazgos y modelo, Lethwood inicia todo un conjunto de estudios sobre el liderazgo en el contexto de la reestructuración escolar, 
uno de los marcos más representativo y de referencia en el campo de la dirección de centros, asociados al liderazgo directivo.

La gran mayoría de los modelos de evaluación de la calidad que se generaron para la autoevaluación, certificación y aseguramiento de la calidad de la gestión, a partir de la década del noventa para las instituciones educativas, se hace dentro del marco de la gestión de la calidad total o también conocido por sus siglas en inglés TQM. Sin embargo, unos están orientados a desarrollar e instalar capacidades de evaluación institucional, mientras que otros se sitúan ya sea en el aseguramiento de la calidad o en prescribir marcos de actuación directiva. Con todo, cada uno de estos modelos hace referencia al factor liderazgo como atributo del director, directora o equipo directivo superior en su capacidad para impulsar más y mejores niveles de logros escolares, a través de instrumentos, tales como la planificación estratégica, el análisis de la información, la comunicación, entre otros.

Debido a la alta complejidad y naturaleza del quehacer pedagógico directivo como al reduccionismo que significa focalizarse en una perspectiva del liderazgo de un modo unipersonal en la función directiva superior, comienza a tomar más fuerza la idea de que el liderazgo en las organizaciones escolares puede ser mejor comprendido y practicado como proceso colectivo de influencias alineadas con el sentido de un proyecto educativo, ya sea por desagregación de roles directivos (direcciones, coordinaciones, profesorado, líderes estudiantiles) o por agregación, a través de los equipos directivos. Asimismo, en una perspectiva más interactiva, dinámica y holística, en que el nivel de confianza organizacional alcanza su más alto sentido, la práctica del liderazgo distribuido emerge como un atributo de la cultura organizacional, en que tanto liderazgos formales como informales interactúan naturalmente e influyen, según situación y niveles de competencias, con el objeto de dinamizar el centro educativo hacia su mejora sustentable. De este modo, como afirman Grubb y Flessa (2009), pueden darse fórmulas junto al coliderazgo o direcciones rotativas o también escuelas sin direccions, donde el personal docente se reparte funciones directivas.

\section{ReFERENCIAS}

Alvariño, C., Arzola, S., Brunner, J. J., Recart. M. O. y Vizcarra, R. (2000). Gestión escolar: Un estado del arte de la literatura. Revista Paideia, 29, 15-43.

Anderson, M. (2012). The Struggle for Collective Leadership: Thinking and Practice in a Multi-campus School Setting [La lucha por el liderazgo colectivo: Pensamiento y práctica en una escuela multi-campus Ajuste]. Educational Management Administration y Leadership, 40(3), 328-342. doi: https://doi.org/10.1177/1741143212436955

Andrews, R., Soder, R. y Jacoby, D. (1986). Principal roles, other in-school variables, and academic achievement by ethnicity and SES [Papeles principales, otras variables dentro de la escuela, y el rendimiento académico por grupo étnico y SES]. Ponencia presentada en la Conferencia anual de la American Educational Research Association, San Francisco.

Bass, B. y Avolio, B. (1994). Evaluate the impact of transformational leadership training at individual, group, organizational and community levels [Evaluar el impacto de la capacitación en liderazgo transformacional en los niveles individual, grupal, organizacional y comunitario]. New York: Binghamton University.

Bennet, N., Wise, C., Woods, P. y Harvey, J. (2003). Distributed Leadership: A review of literature [Liderazgo Distribuido: Una revisión de la literatura]. London: National College of School Leadership.

Bennis, W. (2000). El fin del liderazgo. Harvard Deusto Business Review, 95, 4-12.

Calvo de Mora, J. (2011). Aspectos críticos del liderazgo institucional en la educación. Revista Electrónica Actualidades Investigativas en Educación 11(2),1-29. doi: http://dx.doi.org/10.15517/aie.v11i2.10210

Coleman, J. S., Campbell, E., Hobson, C., McPartland, J., Mood, A., Weinfeld, F. y York, R. (1966). Equality of educational opportunity [Igualdad de oportunidades educativas]. Washington, U.S. Government Printing Office. 
Conger, J. A., y Pearce, C. L. (2003). A landscape of opportunities [Un paisaje de oportunidades]. In C. L. Pearce y J. A. Conger (Eds.), Shared leadership: Reframing the hows and whys of leadership (pp. 285-303). Thousand Oaks, CA: Sage. doi: https://doi.org/10.4135/9781452229539.n14

Crawford, M. (2012). Solo and Distributed Leadership: Definitions and Dilemmas [Solo y distribuidos. Liderazgo: Definiciones y dilemas]. Educational Management Administration and Leadership, 40(5), 610-620. doi: https://doi.org/10.1177/1741143212451175

Cuadra, G. (2014). La gestión escolar y los desafíos en la formación de directivos. Recuperado de http:// mailing.uahurtado.cl/cuaderno_educacion_63/pdf/escuela/escuela_gestion_escolar.pdf

Day, C. (2013). Prácticas exitosas de liderazgo educativo desde una perspectiva comparada. Bilbao: Ediciones Mensajero.

Elmore, R. (2010). Mejorando la escuela desde la sala de clases. Santiago de Chile: Fundación Chile-CAP.

Engeström, Y. (2001). Los estudios evolutivos del trabajo como punto de referencia de la teoría de la actividad: El caso de la práctica médica de la asistencia básica. En S. Chaiklin y J. Lave (Comps.), Estudiar las prácticas: Perspectivas sobre actividad y contexto (pp. 78-118). Buenos Aires: Amorrortu Editores.

Grift, W. V. D. (1990). Educational leadership and academic achievement in elementary education [Liderazgo educativo y el rendimiento académico en la educación primaria]. School Effectiveness and School Improvement, 1(1), 26-40. doi: https://doi.org/10.1080/0924345900010104

Gronn, P. (2002). Distributed leadership as a unit of analysis [Liderazgo distribuido como una unidad de análisis]. Leadership Quarterly, 13(4), 423-451. doi: https://doi.org/10.1016/S1048-9843(02)00120-0

Grubb, W. N. y Flessa, J. J. (2009). A job too big for one" Multiple principals and other nontraditional approaches to school leadership. En K. Leithwood, B. Mascall y T. Strauss (Coords.), Distributed leadership according to the evidence (pp. 137-164). Nueva York: Routledge.

Hallinger, P. (2008). Methodologies for studying school leadership: A review of 25 years of research using the principal instructional management rating scale [Metodologías para el estudio de liderazgo de la escuela : Una revisión de 25 años de investigación utilizando la escala de calificación de gestión de instrucción director]. Ponencia presentada en la Conferencia anual de la American Educational Research Association, New York.

Hallinger, P. (2012). School Leadership that Makes a Difference: Lessons from 30 Years of International Research [Liderazgo de la escuela que hace: Lecciones de diferencia a partir de 30 años de investigación internacional]. Roma: Ministry of Education.

Harris, A. (2008). Distributed leadership through the looking glass. [El liderazgo distribuido a través del espejo] Journal of Educational Administration, 22(1), 31-34. doi: 10.1177/0892020607085623

Harris, A. (2009). Distributed leadership in schools: Developing leader tomorrow [El liderazgo distribuido en las escuelas: Desarrollo de líder de mañana]. Londres: Routledge y Falmer press. doi: https:// doi.org/10.1007/978-1-4020-9737-9

Harris, A. (2012a). Distributed leadership: implications for the role of the principal [Distribución de liderazgo: Implicaciones para el rol del director] Journal of Management Development, 31(1), 7-17. doi: https:// doi.org/10.1108/02621711211190961

Harris, A. (2012b). Liderazgo y desarrollo de capacidades en la escuela. Santiago de Chile: Centro de innovación en Educación de Fundación Chile.

Hutchins, E. (2000). Distributed cognition [Cognición distribuida]. En N. Smelser y P. Baltes (Eds.), International Encyclopedia of the Social and Behavioral Sciences. Estados Unidos de Norteamérica: Elsevier Science.

Immegart, G. (1996). Dirección participativa: Realidades prácticas, estrategias y éticas. Bilbao: ICE. Universidad de Deusto.

Juste, R. P., López, F., Peralta, M y Municio, P. (2001). Hacia una educación de calidad: Gestión, instrumentos y evaluación. Madrid: Narcea Ediciones.

Lashway, L. (1995). Can Instructional Leaders Be Facilitative Leaders? [¿Pueden los líderes de instrucción ser líderes facilitadores?]. ERIC Digest, 98. Recuperado de https://www.ericdigests.org/1996-1/leaders.htm 
Leithwood, K. (1994). Leadership for School Restructuring. [ Liderazgo para la reestructuración escolar] Educational Administration Quarterly, 30(4), 498-518. doi: https://doi.org/10.1177/0013161x94030004006

Leithwood, K. (2009). Efectos del liderazgo transformacional sobre las condiciones organizacionales y el compromiso de los alumnos con la escuela. En ¿¿Cómo liderar nuestras escuelas? Aportes desde la investigación (pp.35-54). Santiago: Salesianos Impresores.

Leithwood, K. A. y Montgomery, D. J. (1986). Improving principal effectiveness: The principal profile [Mejorar la eficacia del director: El perfil de director]. Ontario: Inst for Studies in Education.

Leithwood, K. y Rhiel, C. (2005). What we know about successful school leadership [Lo que sabemos sobre el liderazgo escolar exitoso]. En W. Firestone y Rhiel (Eds), A new agenda: directions from research on educational leadership (pp. 22-47). New York: Teachers College Press.

Longo, F. (2008). Liderazgo distribuido, un elemento crítico para promover la innovación. Capital Humano, 226, $84-91$.

López, P. (2010). El componente liderazgo en la validación de un modelo de gestión escolar hacia la calidad. Educação e Pesquisa, 36(3), 779-794. doi: https://doi.org/10.1590/S1517-97022010000300009

López, P. (2013). Fundamentos epistemológicos del liderazgo distribuido: El caso de la investigación en educación. Cinta Moebio, 47, 83-94. doi: https://doi.org/10.4067/S0717-554X2013000200003

López, J., García, E., Oliva, N., Moreta, B. y Bellerín, A. (2014). El liderazgo escolar a través del análisis de la actividad diaria de los directores. REICE. Revista Iberoamericana sobre Calidad, Eficacia y Cambio en Educación, 12(5), 61-7.

Macbeath, J. (2011). Liderar el aprendizaje dentro y fuera de la escuela. Área de Educación. Fundación Chile. Santiago: Salesianos.

Maureira, O. (2004). Liderazgo factor de eficacia escolar, hacia un modelo causal. Revista Electrónica Iberoamericana sobre Calidad, Eficacia y Cambio en Educación. 2(1), 1-20. Recuperado de http://www.ice.deusto.es/rinace/ reice/vol2n1/Maureira.pdf

Maureira, O. (2007). Liderazgo para la eficacia escolar: Una estrategia para la calidad. Santiago de Chile: Ediciones UCSH.

Maureira, O., Moforte, C., y González, G. (2014). Más liderazgo distribuido y menos liderazgo directivo: Nuevas perspectivas para caracterizar procesos de influencia en los centros escolares. Perfiles educativos, 36(146), 134-153. doi: https://doi.org/10.1016/S0185-2698(14)70132-1

Ministerio de Educación de la República de Chile (Mineduc). (2015). Marco para la buena dirección y liderazgo. Santiago de Chile: Maval. Recuperado de http://portales.mineduc.cl/usuarios/cpeip/ doc/201511131613560.MBD\&LE_2015.pdf

Murillo, F. J. (2006). Una dirección escolar para el cambio: Del Liderazgo transformacional al liderazgo distribuido. Revista Electrónica Iberoamericana sobre Calidad, Eficacia y Cambio en Educación, 4(4e), 11-24. Recuperado de http://www.redalyc.org/pdf/551/55140403.pdf

Murphy, J. A., y Hallinger, P. (1984). Policy analysis at the local level: A framework for expanded investigation [El análisis de políticas a nivel local: Un marco para la investigación ampliada]. Educational Evaluation and Policy Analysis, 6(1), 5-13. doi: https://doi.org/10.3102/01623737006001005

OCDE. (2009). Informe TALIS: La creación de entornos eficaces de enseñanza y aprendizaje. Síntesis de los primeros resultados. Recuperado de http://168.255.201.80/TALIS2009/Sintesis_TALIS_Internacional.pdf

Riveros-Barrera, A. (2012). La distribución del liderazgo como estrategia de mejoramiento institucional. Educación y Educadores, 15(3), 289-301. doi: https://doi.org/10.5294/edu.2012.15.2.7

Robinson, V., Hohepa, M. y Lloyd, C. (2009). School leadership and student outcomes: Identifying what works and why: Best evidence synthesis iteration (BES) [Resultados de liderazgo escolar y estudiantil : Identificar lo que funciona y por qué: La mejor evidencia de síntesis de iteración]. Wellington, New Zeland: Ministry of Education.

Senge, P., Ross, R., Smith, B., Roberts, C. H., y Kleiner, A. (1995). La quinta disciplina en la práctica. Estrategias para construir la organización abierta al aprendizaje. Madrid: Granica. 
Sergiovanni, T.J. (1984). Leadership and excellence in schooling [Liderazgo y excelencia en la educación]. Educational leadership, 41(5), 4-13.

Spillane, J. (2006). Distributed leadership [Liderazgo distribuido]. San Francisco: Jossey-Bass.

Spillane, J. P., y Healey, K. (2010). Conceptualizing school leadership and management from a distributed perspective: An exploration of some study operations and measures. [La conceptualización de liderazgo y gestión escolar desde una perspectiva distribuida: Una exploración de algunas operaciones de estudio y medidas]. The Elementary School Journal, 111(2), 253-281. doi: https://doi.org/10.1086/656300

Spillane, J. P., Camburn, E. M., Pustejovsky, J., Stitziel, A., y Lewis, G. (2008). Taking a distributed perspective: Epistemological and methodological tradeoffs in operationalizing the leader-plus aspect [Tomando una perspectiva distribuida: Supuestos epistemológicos y metodológicos en la práctica del aspecto - Leader Más-]. Journal of Educational Administration, 46(2), 189-213. doi: https://doi.org/10.1108/09578230810863262

Spillane, J., Diamond, J. y Jita, L. (2003). Leading instruction: the distribution of leadership for instruction [Instrucción de ejecución: La distribución del liderazgo para la instrucción]. Journal of Curriculum Studies, 35(5), 533-543. doi: https://doi.org/10.1080/0022027021000041972

Spillane, J., Halverson, R. y Diamond, J. (2004). Towards a theory of leadership practice: a distributed perspective [Hacia una teoría de la práctica del liderazgo: Una perspectiva distribuida]. Journal of Curriculum Studies, 36(1), 3-34. doi: https://doi.org/10.1080/0022027032000106726

Vera, L. (2016). Liderazgo decisional distribuido, condiciones y razones asociadas a su mayor o menor recurrencia en establecimientos municipales que implementan planes de mejoramiento educativo. (Tesis doctoral inédita). Universidad Playa Ancha. Valparaíso, Chile.

Villa, A. (2009). El perfil del liderazgo en los centros educativos de enseñanzas medias de Guipúzcoa. San Sebastián: Diputación Foral de Guipúzcoa.

Villanova, R., Gauthier, W., Proctor, P., y Shoemaker, J. (1981). The Connecticut school effectiveness questionnaire [El cuestionario eficacia escolar de Connecticut]. Hartford, CT: Bureau of School Improvement, Connecticut State Department of Education.

Weinstein, J. y Muñoz, G. (2012). ¿Qué sabemos de los directores de escuela en Chile?. Santiago de Chile: Centro de Estudios de Políticas en Educación.

\section{Notas}

[1] La revisión descrita fue realizada en parte gracias al apoyo del Fondo Nacional de Ciencia y Tecnología de Chile (FONDECYT) a través del proyecto N..$^{\circ} 1130161$ : Liderazgo distribuido en instituciones de enseñanza primaria: sus antecedentes y efectos en el incremento de la mejora escolar.

\section{BY-NC-ND}

